\title{
Stress testing the vector-boson-fusion approximation in multijet final states
}

\author{
Francisco Campanario \\ Theory Division, IFIC, University of Valencia-CSIC, E-46980 Paterna, Valencia, Spain \\ and Institute for Theoretical Physics, Karlsruhe Institute of Technology, 76131 Karlsruhe, Germany \\ Terrance M. Figy \\ Department of Mathematics, Statistics, and Physics, Wichita State University, \\ Wichita, Kansas 67260, USA \\ Simon Plätzer \\ Particle Physics, Faculty of Physics, University of Vienna, 1090 Vienna, Austria \\ Michael Rauch \\ Institute for Theoretical Physics, Karlsruhe Institute of Technology, 76131 Karlsruhe, Germany \\ Peter Schichtel \\ German Research Center for Artificial Intelligence (DFKI), D-67663 Kaiserslautern, Germany \\ and IAV Automotive Engineering, D-67663 Kaiserslautern, Germany \\ Malin Sjödahl \\ Department of Astronomy and Theoretical Physics, Lund University, 22100 Lund, Sweden
}

(Received 7 March 2018; published 22 August 2018)

\begin{abstract}
We consider electroweak Higgs plus three jets production at NLO QCD beyond strict VBF acceptance cuts. We investigate, for the first time, how accurate the VBF approximation is in these regions and within perturbative uncertainties by a detailed comparison of full and approximate calculations. We find that a rapidity gap between the tagging jets guarantees a good approximation, while an invariant mass cut alone is not sufficient, which needs to be confronted with experimental choices. We also find that a significant part of the QCD corrections can be attributed to Higgs-Strahlungs-type topologies.
\end{abstract}

DOI: $10.1103 /$ PhysRevD.98.033003

\section{INTRODUCTION}

In 2012, both the ATLAS [1] and CMS [2] Collaborations announced the discovery of a new boson in the mass range of $125-126 \mathrm{GeV}[3,4]$. There were indications that this new particle behaved very similarly to the Higgs boson of the standard model [5-12] and recently, the ATLAS and CMS Collaborations reported the standard model hypothesis to be consistent with data in a combined analysis of LHC proton-proton collision data at $\sqrt{s}=7$ and $8 \mathrm{TeV}$ [13]. The vector-boson fusion (VBF) signature [14-29] is among the most important production channels of the Higgs boson in the ongoing run of the Large Hadron Collider (LHC). For a Higgs boson accompanied by at least

Published by the American Physical Society under the terms of the Creative Commons Attribution 4.0 International license. Further distribution of this work must maintain attribution to the author(s) and the published article's title, journal citation, and DOI. Funded by SCOAP . two jets in the final state, the underlying production processes allow for both spacelike, $t$-channel, exchange of weak gauge bosons producing a Higgs boson, as well as timelike Higgs-Strahlung type topologies of associated production with a vector boson which decays into a quark-antiquark pair, cf. Fig 1. All of the contributing diagrams do interfere, and the $\mathrm{VBF}$ region is usually referred to as a phase space region in which one expects the $t$-channel diagrams to dominate, with timelike $s$-channel and interference effects broadly suppressed. In the $\mathrm{VBF}$ region, one requires two highly energetic jets, well separated in rapidity and with the Higgs boson decay products located in the central detector region and possibly in between the two jets. Additionally, a veto on central QCD activity is sometimes applied to enrich the contribution of the color singlet vector boson exchange [30-35].

Theoretical predictions in this region often employ the so-called VBF approximation, where only the $t$-channel topology is kept and $s$-channel contributions as well as interference effects between different topologies are 

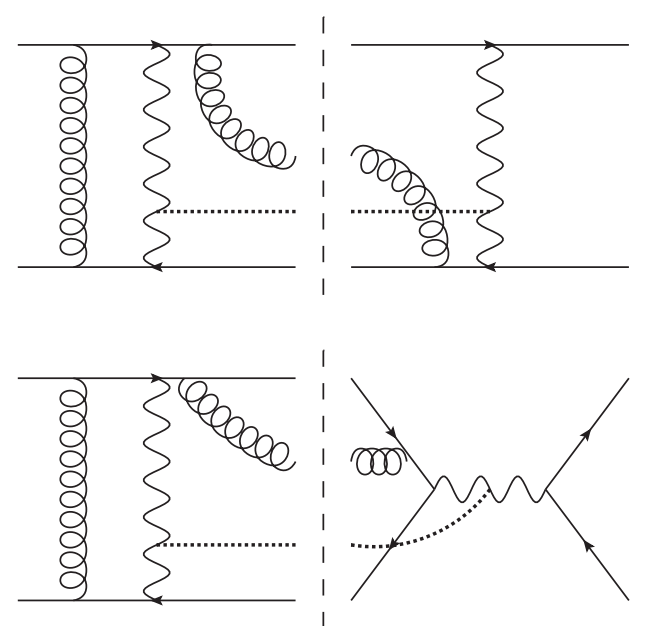

FIG. 1. In the VBF region, interferences among certain $t$-channel topologies (top diagram) as well as $t / s$-channel (lower diagram, and $t / u$-channel, not depicted) interferences are neglected.

neglected. Figure 1 gives an example of some of the contributions which are typically not considered. Formally, this corresponds to the approximation that the constituents of the two incoming protons belong to two different, but otherwise identical, copies of the color gauge group $S U(3)$. Recent experimental analyses do not implement selection criteria for the VBF region as tight as originally envisaged [14-20], and rely on a multitude of multivariate analysis techniques instead [36]. While for the Higgs plus two jet case the validity of the VBF approximation has been confirmed within a tight selection [21,22], essentially nothing is known quantitatively for additional radiation as relevant to the veto on central jets (CJV), or virtually any observable exploiting properties of the radiation pattern of the underlying electroweak production process.

Next-to-leading order corrections in quantum chromodynamics (QCD) to the three jet process are available without any approximation [37] as a plugin to the MATCHBOX framework [38] of the HERWIG 7 event generator [39-41], and can be compared to calculations based on the VBF approximation [42] as implemented in the VBFNLO program [43-45]. In this article, we quantify the reliability of the VBF approximation, i.e., the neglection of the diagrams which are not of the VBF $t$-channel topology along with interference effects with $u$-channel topologies.

\section{OUTLINE OF THE CALCULATION}

We use the HERWIG 7 event generator in its recent release 7.1.2 [40,41], together with HJETS++ 1.1 [46] to provide the amplitudes for electroweak Higgs boson plus jets production. The color structure is treated using ColorFull [47] and the loop integrals are computed following Ref. [48]. For the VBF approximation we rely on the approximate calculation provided by VBFNLO version 3.0 beta 5 . Both calculations have recently also been interfaced to parton showers using different matching paradigms, for a dedicated comparison see $[49,50]$. The one-loop matrix elements of HJETS++ and VBFNLO have been cross-checked against those of MadLoOP [51], GoSAm 2.0 [52], and OpenLoops [53] at the level of phase space points.

We have ensured that both programs run with the same set of electroweak parameters in a $G_{\mu}$ scheme with input parameters $G_{F}=1.16637 \times 10^{-5} \mathrm{GeV}^{-2}$, $M_{Z}=91.1876 \mathrm{GeV}$ and $M_{W}=80.403 \mathrm{GeV}$. The electromagnetic coupling constant and the weak-mixing angle are calculated via tree level relations. We take the Higgs boson as stable, with a mass fixed to $m_{H}=125.7 \mathrm{GeV}$. The widths of the bosons are fixed to $\Gamma_{Z}=2.4952 \mathrm{GeV}$ and $\Gamma_{W}=2.141 \mathrm{GeV}$. We consider proton-proton collissions at $13 \mathrm{TeV}$ center of mass energy and employ a four-flavor scheme with the MMHT $201468 \%$ C.L. PDF set at NLO [54] with a two-loop running $\alpha_{s}$ set at $\alpha_{s}\left(M_{Z}\right)=0.12$ with $m_{c}=1.4 \mathrm{GeV}$ and $m_{b}=4.75 \mathrm{GeV}$.

We select jets using the anti- $k_{\perp}$ algorithm as implemented in the FASTJET library $[55,56]$, with a cone radius of $R=0.4$, and accept jets ordered in transverse momentum, with a transverse momentum $p_{\perp, j}>30 \mathrm{GeV}$ inside a rapidity range of $\left|y_{j}\right|<4$.4. No restrictions are applied to the Higgs boson acceptance, nor any other jet kinematic variable. We then use this baseline acceptance to scan through possible cuts. Specifically, we consider tagging jet acceptances in intervals of the leading dijet invariant mass $m_{12}=\sqrt{\left(p_{1}+p_{2}\right)^{2}}$, and the leading jet pair rapidity separation $\Delta y_{12}=\left|y_{1}-y_{2}\right|$,

$$
\begin{aligned}
m_{12} & >m_{12}^{\text {cut }} \in\{0,100,200,300,400,500,600\} \mathrm{GeV}, \\
\Delta y_{12} & >\Delta^{\text {cut }} y_{12} \in\{0,1,2,3\} .
\end{aligned}
$$

The central renormalization, $\mu_{R}$, and factorization, $\mu_{F}$, scales are chosen to be $H_{\perp}$ (jets), which we here define as

$$
H_{\perp}=\frac{1}{2} \sum_{\substack{i \text { jets } \\\left(p_{\perp}>15 \mathrm{GeV}\right)}} p_{\perp, i},
$$

where jets are clustered as outlined above, and only subject to a reduced transverse momentum cut with $q_{\perp}=15 \mathrm{GeV}$, which is required to make the scale definition infrared and collinear safe. Note that the jet cuts in the scale definition are more inclusive than the analysis jet cuts.

The full calculation contains Higgs-Strahlung (VH) topologies, which interfere with the possible VBF-type diagrams, as depicted in Fig. 1. While we expect these contributions not to be relevant within tight VBF selection criteria, they might well contribute when relaxing these constraints and as such yield a biased view on quantifying the accuracy of the VBF approximation. Simulations used by experimentalists also use a mix of $\mathrm{VH}$ and VBF processes, but without interferences and without the pentagon (Fig. 1) and hexagon topologies, implying that 
biased simulations go into experimental decisions and interpretation.

To work as closely as possible to the simulations used by experimentalists we remove the $\mathrm{VH}$ contributions by applying a resonance-veto on any single- and multijet masses in the neighborhood of the $W^{ \pm}$and $Z$ masses, i.e.,

$$
m_{V}-\delta m_{V}<m_{\mathrm{jets}}<m_{V}+\delta m_{V}
$$

with $V=W^{ \pm}, Z$, choosing $\delta m_{Z}=\delta m_{W}=5 \mathrm{GeV}$. We stress that such an operational definition of eliminating those contributions not taken into account in an approximate setup is required, as neglecting individual diagrams or interferences in the full calculation will result in a nongauge-invariant prediction, unless one imposes the full VBF approximation. We discuss results both with, and without such a cut applied. All analyses have been performed using a dedicated analysis implemented in RIVET [57].

\section{IMPACT OF QCD CORRECTIONS}

For the inclusive selection, QCD corrections have been found to be moderate for Higgs kinematics but significant for third jet properties, specifically in the high- $p_{\perp, 3}$ regime [37]. The approximate calculation suggests small corrections with a significant reduction in scale uncertainty. Prior

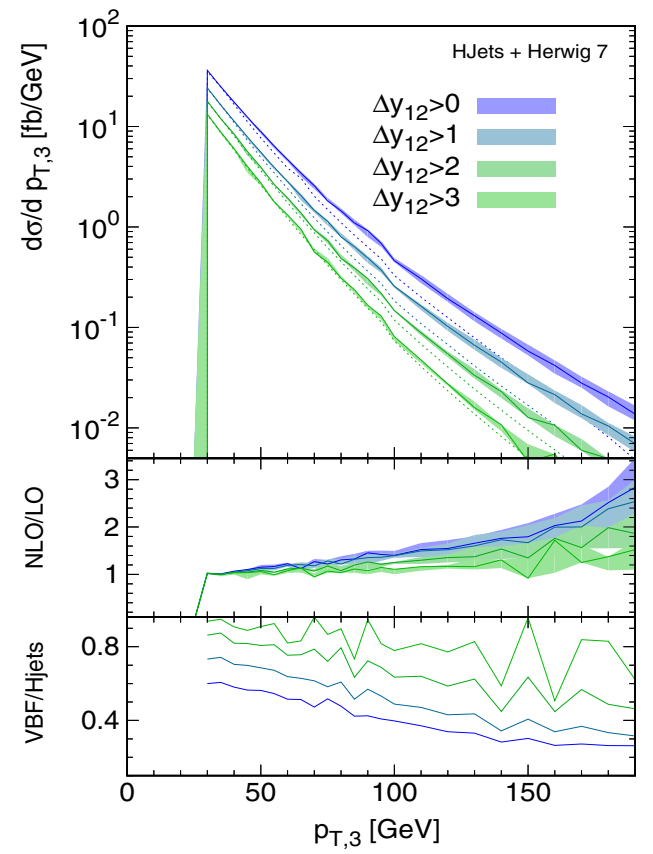

FIG. 2. QCD corrections on the third jet transverse momentum $p_{T, 3}$ spectrum for the full and approximate calculations for $m_{12}^{\text {cut }}=0 \mathrm{GeV}$ and several choices of $\Delta^{\text {cut }} y_{12}$ (see Eq. (1): the predictions for the full calculation, the corresponding differential $K$-factor and the ratio of the approximated over the full calculation are plotted in the top, middle and bottom panels, respectively. We show LO results (dotted lines), NLO results (solid lines) with scale variations (light bands). to studying the differences between the exact and the approximate calculations, we have investigated the effect of QCD corrections subject to tight VBF cuts, implemented by requesting a rapidity gap of $\Delta y_{12}>3$ and a invariant mass of $m_{12}>600 \mathrm{GeV}$ for two tagged jets. We find that NLO corrections in the VBF region are small, and the full and approximate calculations are in reasonable agreement within $3 \%$, with scale variations increasing by $8 \%$ upon vetoing on resonant structures.

Shown in the top panel of Fig. 2 is the NLO (solid) and LO (dotted) transverse momentum spectrum $p_{\perp, 3}$ of the third jet for the full calculation with $m_{12}^{\text {cut }}=0 \mathrm{GeV}$, no resonance-veto cut applied and several choices of $\Delta^{\text {cut }} y_{12}$. The bands represent the NLO scale uncertainty in the range $H_{\perp} / 2 \leq \mu_{F}=\mu_{R} \leq 2 H_{\perp}$.

In all of the figures displaying differential cross sections, the middle panel shows differential $K$-factors, defined as $\mathrm{d} \sigma_{\mathrm{NLO}} / \mathrm{d} \sigma_{\mathrm{LO}}$, where the bands reflect the NLO scale variations with respect to the leading order calculation fixed at the central scale. The increased $K$-factor in the high transverse momentum region can be traced back to $V H+1$ jet type events, and the resonance veto has the effect of reducing the corrections down to values of 1.4 in the high transverse momentum region for the inclusive selection cuts $\left(\Delta y_{12}>0\right)$ (not shown). In the lower panel of Fig. 2, the ratio of the approximate to the full result is plotted. Differences of order $50 \%$ and more are visible when no rapidity separation is required. However, as the rapidity gap

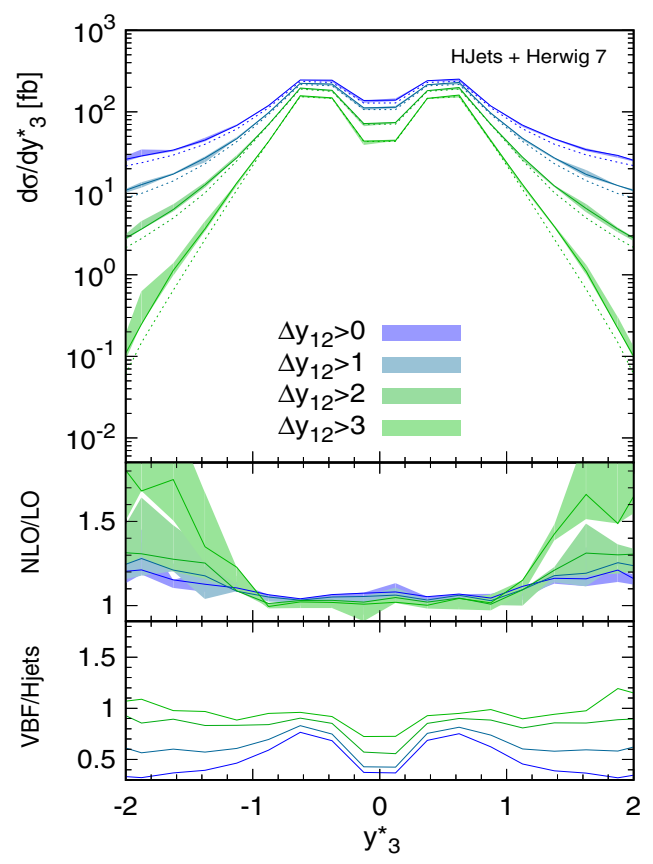

FIG. 3. The normalized centralized rapidity $y_{3}^{\star}$ distribution of the third jet for various leading jet separation rapidity cuts. The upper, middle and lower panel show, respectively, the full NLO and LO calculation (solid and dotted lines), the $K$-factor and the ratio of the VBF-approximation to the full NLO result. 
increases, the large $K$-factor in the transverse momentum spectrum for the full calculation is reduced (cf. middle panel), and the full and approximate results display differences at the $20 \%$ level in the bulk of the corrections for $\Delta y_{12}>0$ (few to ten percent up to $p_{T, 3}<100 \mathrm{GeV}$ with a resonance-cut applied), and increase up to $50 \%$ (30\% with a resonance-cut) in the range shown.

In the upper panel in Fig. 3, we consider the normalized centralized rapidity distribution of the third jet $y_{3}^{*}=$ $\left(y_{3}-\frac{1}{2}\left(y_{1}+y_{2}\right)\right) /\left|y_{1}-y_{2}\right|$ without resonance-veto cut for the full NLO calculation as solid lines with scale uncertainty error bands, as well as the LO result (dotted lines). QCD corrections tend to increase for high rapidity separations $\Delta y_{12}$. We find a clear improvement of the VBFapproximation for high rapidity separations, whereas it will clearly underestimate the full result if no rapidity separation is required. This observation even holds when resonancecuts are applied (not shown) with differences in the central and extreme regions of the plot of about $40 \%$ for the $\Delta y_{12}>0$ curve.

\section{EFFECTS OF $\Delta y_{12}$ AND $m_{12}$ SELECTIONS}

Figure 4 depicts the rapidity separation of the two leading jets for several choices of $m_{12}^{\text {cut }}$ (left) and the dijet invariant mass of the leading two jets for several choices of $\Delta^{\text {cut }} y_{12}$ (right). The resonance-veto described in Eq. (3) has been enforced in the event selection contributing to these observables. We again compare NLO results (solid lines) with scale uncertainty error bands for the full calculation as well as LO

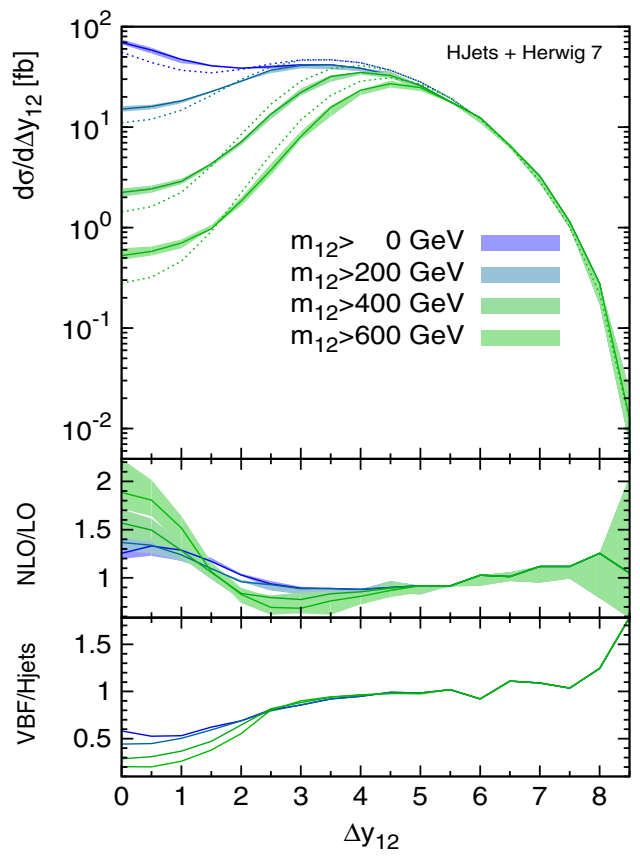

results (dotted lines). NLO corrections ( $K$-factors shown in the middle panels) can reach the $40 \%$ level for rapidity separation values $\leq 1$ and dijet invariant masses of $100 \mathrm{GeV}$. Increasing the invariant mass cut $m_{12}$ beyond approximately $100 \mathrm{GeV}$ results in smaller NLO corrections for all rapidity separations (as seen in the right plot of Fig. 4). The quality of the VBF approximation is shown in the lower ratio plots. Deviations of the order of several ten per cent are visible for small rapidity separations and/or small dijet invariant masses. Increasing values of $\Delta^{\text {cut }} y_{12}$ result in better agreement between the full and approximate results (left). However, the full and approximate calculations are not guaranteed to agree in the presence of a cut on the dijet invariant mass alone (right plot with $\Delta y>0$ ).

While we have so far only presented a few observables to quantify the impact of QCD corrections and the validity of the VBF approximation, the calculation we performed has actually involved a large number of observables sensitive to the kinematic distribution of the third jet as well as dedicated VBF observables. In order to quantify the quality of the approximation across the whole set of these observables, we consider a metric inspired by a statistical test and calculate

$$
\chi^{2}=\frac{1}{N_{\text {bins }}} \sum_{\text {bins } i} \frac{\left(\sigma_{i, \mathrm{HJets}}-\sigma_{i, \mathrm{VBF}}\right)^{2}}{\max \left(\delta_{\mu} \sigma_{i}^{2}, \delta_{\mathrm{stat}} \sigma_{i}^{2}\right)}
$$

where we consider the maximum of scale variation $\delta_{\mu} \sigma_{i}$ or statistical deviation $\delta_{\text {stat }} \sigma_{i}$ per bin to set the scale of fluctuations within which we want to measure agreement.

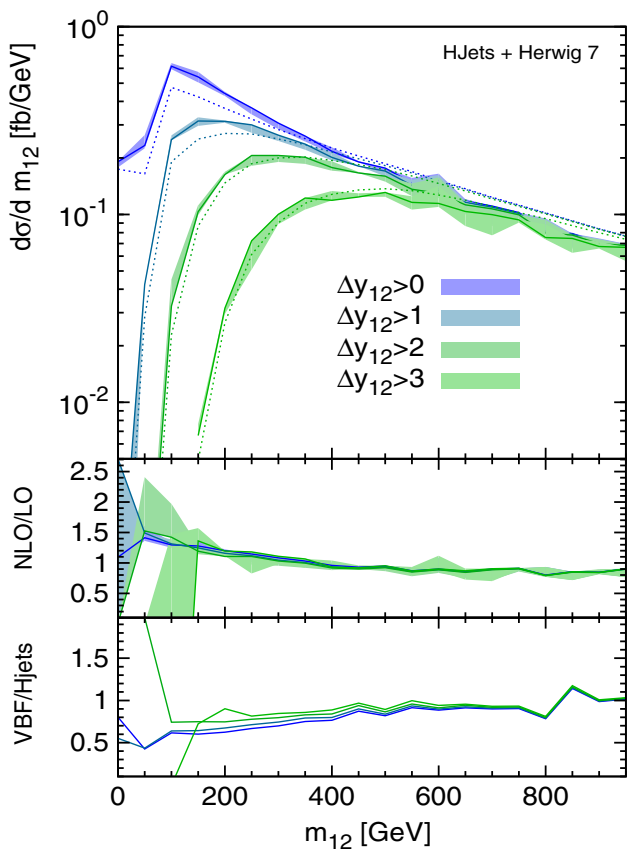

FIG. 4. The rapidity separation $\Delta y_{12}$ of the leading two jets, for different cuts on their invariant mass (left) and the jet-jet invariant mass $m_{12}$ as a function of the rapidity gap requirement (right). We compare NLO QCD predictions in the full calculation (solid) to the approximate results (dashed). 

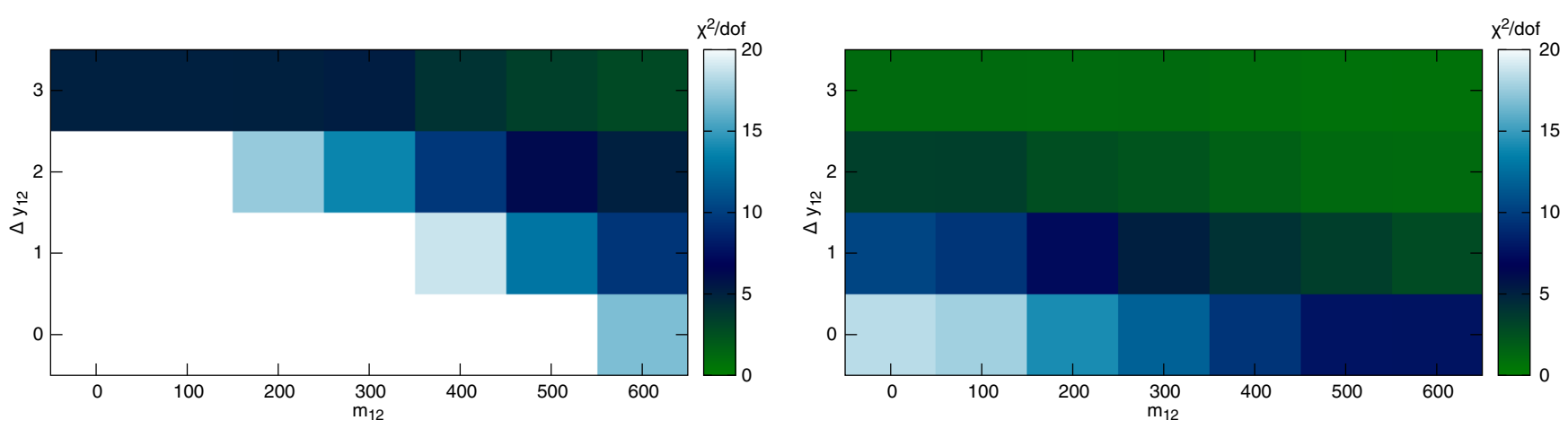

FIG. 5. Compatibility of the approximate predictions to the full calculation as a function of VBF acceptance cuts without (left) and with (right) applying vetos on Higgs-Strahlung-type contributions. We calculate goodness-of-fit measures based on the scale variation uncertainty for a range of observables relevant to the typical VBF kinematics. For the degrees of freedom we count only nonzero bins. Their number ranges from 101 to 129.

This is important to check the compatibility of both calculations within their intrinsic uncertainties, or within statistical uncertainties where not avoidable due to the presence of a small cross section. The results are presented in Fig. 5, where we include $p_{T, 3}, y_{3}^{\star}, y_{h}^{\star}, \Delta y_{h, 12}, \Delta \phi_{h, 12}$, and $m_{123}$ in the goodness-of-fit calculation as a function of $\Delta^{\text {cut }} y_{12}$ and $m_{12}^{\text {cut }}$ without (left column) and with (right column) the resonance-veto on the Higgs-Strahlung-type events Eq. (3). We can clearly observe that the VBF approximation can be considered valid only for dijet invariant mass cuts above $500 \mathrm{GeV}$ and for rapidity gaps above 2. It would seem as if the VBF cuts do not remove the $H V j$ events effectively even in tight VBF selections. In contrast, for the resonance-veto case agreement starts near $m_{12}=500 \mathrm{GeV}$ and a rapidity gap of 0 , however only a rapidity gap cut of at least 2 units guarantees decent agreement between the full and approximate calculations.

\section{CONCLUSIONS AND OUTLOOK}

In this article, we have addressed the quality of the vector boson fusion approximation in three jet events by comparing full and approximate calculations at NLO QCD. While moderate rapidity separation cuts guarantee convergence at the percent level, large dijet invariant mass cuts are not sufficient to achieve the same accuracy. This important information should be taken into account in experimental analyses. In addition, we have shown that the NLO QCD corrections of the full calculation can reach a factor of 3 and are consistent with Higgs-Strahlung $V H j$ contributions.

\section{ACKNOWLEDGMENTS}

F. C. acknowledges financial support by the Generalitat Valenciana, Spanish Government and ERDF funds from the European Commission (Grants No. RYC-2014-16061, No. SEJI-2017/2017/019, No. FPA2017-84543-P, No. FPA2017-84445-P, and No. SEV-2014-0398). M. R. would like to acknowledge the contribution of the COST Action CA16108. S. P. acknowledges partial support by the COST Action CA16201 PARTICLEFACE. This work used the Extreme Science and Engineering Discovery Environment (XSEDE), which is supported by National Science Foundation Grant No. ACI-1548562 [58]. This work utilized computing resources provided at IPPP, Durham. This work also utilized both the Open Science Grid and Texas Advanced Computing Center (TACC) Stampede 2 to perform event generation runs through allocations TG-TRA150015 and TG-PHY160001. T.F. would like to thank Mats Rynge for assistance with setting up event generation runs on the Open Science Grid, which was made possible through the XSEDE Extended Collaborative Service (ECSS) program. T. F. would like to thank Juan Cruz-Martinez, Jonas Lindert, and Nigel Glover for valuable discussions. M. R. would like to thank Amon Engemann for helpful code comparisons. M. S. was supported by the Swedish Research Council (Contract Nos. 2012-02744 and 2016-05996), and in part by the European Union's Horizon 2020 research and innovation programme (Grant agreement No. 668679).
[1] G. Aad et al. (ATLAS), ATLAS pixel detector electronics and sensors, J. Instrum. 3, P07007 (2008).

[2] S. Chatrchyan et al. (CMS), The CMS experiment at the CERN LHC, J. Instrum. 3, S08004 (2008).
[3] G. Aad et al. (ATLAS), Observation of a new particle in the search for the Standard Model Higgs boson with the ATLAS detector at the LHC, Phys. Lett. B 716, 1 (2012). 
[4] S. Chatrchyan et al. (CMS), Observation of a new boson at a mass of $125 \mathrm{GeV}$ with the CMS experiment at the LHC, Phys. Lett. B 716, 30 (2012).

[5] T. W. B. Kibble, Symmetry breaking in non-abelian gauge theories, Phys. Rev. 155, 1554 (1967).

[6] P. W. Higgs, Spontaneous symmetry breakdown without massless bosons, Phys. Rev. 145, 1156 (1966).

[7] G. S. Guralnik, C. R. Hagen, and T. W. B. Kibble, Global Conservation Laws and Massless Particles, Phys. Rev. Lett. 13, 585 (1964).

[8] F. Englert and R. Brout, Broken Symmetry and the Mass of Gauge Vector Mesons, Phys. Rev. Lett. 13, 321 (1964).

[9] P. W. Higgs, Broken symmetries, massless particles and gauge fields, Phys. Lett. 12, 132 (1964).

[10] P. W. Higgs, Broken Symmetries and the Masses of Gauge Bosons, Phys. Rev. Lett. 13, 508 (1964).

[11] S. Weinberg, A Model of Leptons, Phys. Rev. Lett. 19, 1264 (1967).

[12] S. L. Glashow, Partial-symmetries of weak interactions, Nucl. Phys. 22, 579 (1961).

[13] G. Aad et al. (ATLAS, CMS), Measurements of the Higgs boson production and decay rates and constraints on its couplings from a combined ATLAS and CMS analysis of the LHC pp collision data at $\sqrt{s}=7$ and $8 \mathrm{TeV}$, J. High Energy Phys. 08 (2016) 045.

[14] N. Kauer, T. Plehn, D. L. Rainwater, and D. Zeppenfeld, $H \rightarrow W W$ as the discovery mode for a light Higgs boson, Phys. Lett. B 503, 113 (2001).

[15] D. L. Rainwater, D. Zeppenfeld, and K. Hagiwara, Searching for $H \rightarrow \tau \tau$ in weak boson fusion at the CERN LHC, Phys. Rev. D 59, 014037 (1998).

[16] D. L. Rainwater and D. Zeppenfeld, Searching for $H \rightarrow \gamma \gamma$ in weak boson fusion at the LHC, J. High Energy Phys. 12 (1997) 005.

[17] D. L. Rainwater and D. Zeppenfeld, Observing $H \rightarrow$ $W^{(*)} W^{(*)} \rightarrow e^{ \pm} \mu^{ \pm} p T$ in weak boson fusion with dual forward jet tagging at the CERN LHC, Phys. Rev. D 60, 113004 (1999); Erratum, 61, 099901 (2000).

[18] S.Asai et al., Prospects for the Search for a Standard Model Higgs Boson in ATLAS using Vector Boson Fusion, arXiv: hep-ph/0402254.

[19] K. Cranmer, B. Mellado, W. Quayle, and S. L. Wu, Search for Higgs Bosons Decay $H \rightarrow \gamma \gamma$ using Vector Boson Fusion, arXiv:hep-ph/0401088.

[20] O. J. P. Eboli and D. Zeppenfeld, Observing an invisible Higgs boson, Phys. Lett. B 495, 147 (2000).

[21] M. Ciccolini, A. Denner, and S. Dittmaier, Strong and Electroweak Corrections to the Production of a Higgs Boson +2 Jets via Weak Interactions at the Large Hadron Collider, Phys. Rev. Lett. 99, 161803 (2007).

[22] M. Ciccolini, A. Denner, and S. Dittmaier, Electroweak and QCD corrections to Higgs production via vector-boson fusion at the CERN LHC, Phys. Rev. D 77, 013002 (2008).

[23] P. Bolzoni, F. Maltoni, S.-O. Moch, and M. Zaro, Higgs Boson Production via Vector-Boson Fusion at Next-toNext-to-Leading Order in QCD, Phys. Rev. Lett. 105, 011801 (2010).

[24] P. Bolzoni, F. Maltoni, S.-O. Moch, and M. Zaro, Vector boson fusion at next-to-next-to-leading order in QCD:
Standard model Higgs boson and beyond, Phys. Rev. D 85, 035002 (2012).

[25] M. Cacciari, F. A. Dreyer, A. Karlberg, G. P. Salam, and G. Zanderighi, Fully Differential Vector-Boson-Fusion Higgs Production at Next-to-Next-to-Leading Order, Phys. Rev. Lett. 115, 082002 (2015).

[26] F. A. Dreyer and A. Karlberg, Vector-Boson Fusion Higgs Production at Three Loops in QCD, Phys. Rev. Lett. 117, 072001 (2016).

[27] M. Rauch, Vector-Boson Fusion and Vector-Boson Scattering, arXiv:1610.08420.

[28] M. Rauch and D. Zeppenfeld, Jet clustering dependence of Higgs boson production in vector-boson fusion, Phys. Rev. D 95, 114015 (2017).

[29] J. Cruz-Martinez, T. Gehrmann, E. W. N. Glover, and A. Huss, Second-order QCD effects in Higgs boson production through vector boson fusion, Phys. Lett. B 781, 672 (2018).

[30] V. D. Barger, R. J. N. Phillips, and D. Zeppenfeld, Minijet veto: a tool for the heavy Higgs search at the LHC, Phys. Lett. B 346, 106 (1995).

[31] D. L. Rainwater, R. Szalapski, and D. Zeppenfeld, Probing color-singlet exchange in $Z+2$-jet events at the CERN LHC, Phys. Rev. D 54, 6680 (1996).

[32] V. D. Barger, K.-m. Cheung, T. Han, and D. Zeppenfeld, Single-forward-jet tagging and central-jet vetoing to identify the leptonic WW decay mode of a heavy Higgs boson, Phys. Rev. D 44, 2701 (1991); Erratum, 48, 5444 (1993).

[33] J. R. Forshaw and M. Sjodahl, Soft gluons in Higgs plus two jet production, J. High Energy Phys. 09 (2007) 119.

[34] B. E. Cox, J. R. Forshaw, and A. D. Pilkington, Extracting Higgs boson couplings using a jet veto, Phys. Lett. B 696, 87 (2011).

[35] R. M. D. Delgado, J. R. Forshaw, S. Marzani, and M. H. Seymour, The dijet cross section with a jet veto, J. High Energy Phys. 08 (2011) 157.

[36] ATLAS Collaboration, Measurements of the Higgs boson production cross section via Vector Boson Fusion and associated $\mathrm{W} \mathrm{H}$ production in the $\mathrm{WW}^{*} \rightarrow \ell \nu \ell \nu$ decay mode with the ATLAS detector at $\sqrt{s}=13 \mathrm{TeV}$, CERN Report No. ATLAS- CONF-2016-112, 2016 (unpublished).

[37] F. Campanario, T. M. Figy, S. Plätzer, and M. Sjödahl, Electroweak Higgs Boson Plus Three Jet Production at Next-to-Leading-Order QCD, Phys. Rev. Lett. 111, 211802 (2013).

[38] S. Platzer and S. Gieseke, Dipole showers and automated NLO matching in Herwig++, Eur. Phys. J. C 72, 2187 (2012).

[39] M. Bahr et al., Herwig++ physics and manual, Eur. Phys. J. C 58, 639 (2008).

[40] J. Bellm et al., Herwig 7.0/Herwig++ 3.0 release note, Eur. Phys. J. C 76, 196 (2016).

[41] J. Bellm et al., Herwig 7.1 Release Note, arXiv:1705.06919.

[42] T. Figy, V. Hankele, and D. Zeppenfeld, Dominant next-toleading order QCD corrections to Higgs plus three jet production in vector-boson fusion, J. High Energy Phys. 02 (2008) 076.

[43] K. Arnold et al., VBFNLO: A parton level Monte Carlo for processes with electroweak bosons, Comput. Phys. Commun. 180, 1661 (2009). 
[44] K. Arnold et al., VBFNLO: A parton level Monte Carlo for processes with electroweak bosons-Manual for Version 2.7.0, arXiv:1107.4038.

[45] J. Baglio et al., Release Note - VBFNLO 2.7.0, arXiv: 1404.3940.

[46] http://hjets.hepforge.org, HJets is a tool for calculating cross sections for electroweak Higgs boson production in association of two and three jet production at next-to-leading order in perturbative QCD.

[47] M. Sjodahl, ColorFull: a C++ library for calculations in SU(Nc) color space, Eur. Phys. J. C 75, 236 (2015).

[48] F. Campanario, Towards $p p \rightarrow V V j j$ at NLO QCD: bosonic contributions to triple vector boson production plus jet, J. High Energy Phys. 10 (2011) 070.

[49] D. de Florian et al. (LHC Higgs Cross Section Working Group), Handbook of LHC Higgs Cross Sections: 4. Deciphering the Nature of the Higgs Sector, arXiv: 1610.07922 .

[50] B. Jäger, F. Schissler, and D. Zeppenfeld, Parton-shower effects on Higgs boson production via vector-boson fusion in association with three jets, J. High Energy Phys. 07 (2014) 125.
[51] V. Hirschi, R. Frederix, S. Frixione, M. V. Garzelli, F. Maltoni, and R. Pittau, Automation of one-loop QCD computations, J. High Energy Phys. 05 (2011) 044.

[52] G. Cullen et al., GOSAM-2.0: a tool for automated one-loop calculations within the Standard Model and beyond, Eur. Phys. J. C 74, 3001 (2014).

[53] F. Cascioli, P. Maierhofer, and S. Pozzorini, Scattering Amplitudes with Open Loops, Phys. Rev. Lett. 108, 111601 (2012).

[54] L. A. Harland-Lang, A. D. Martin, P. Motylinski, and R. Thorne, Parton distributions in the LHC era: MMHT 2014 PDFs, Eur. Phys. J. C 75, 204 (2015).

[55] M. Cacciari, G. P. Salam, and G. Soyez, FastJet user manual, Eur. Phys. J. C 72, 1896 (2012).

[56] M. Cacciari and G. P. Salam, Dispelling the $N^{3}$ myth for the $K_{t}$ jet-finder, arXiv:hep-ph/0512210.

[57] A. Buckley, J. Butterworth, D. Grellscheid, H. Hoeth, L. Lönnblad, J. Monk, H. Schulz, and F. Siegert, Rivet user manual, Comput. Phys. Commun. 184, 2803 (2013).

[58] J. Towns et al., XSEDE: Accelerating Scientific Discovery, Comput. Sci. Eng. 16, 62 (2014). 